\title{
Raman Amplifier Based on Amorphous Silicon Nanoparticles
}

\author{
M. A. Ferrara, ${ }^{1}$ I. Rendina, ${ }^{1}$ S. N. Basu, ${ }^{2}$ L. Dal Negro, ${ }^{3}$ and L. Sirleto ${ }^{1}$ \\ ${ }^{1}$ Institute for Microelectronics and Microsystems, National Research Council, Via P. Castellino 111, 80131 Napoli, Italy \\ ${ }^{2}$ Division of Materials Science and Engineering, Boston University, 15 Saint Mary's Street, Brookline, \\ MA 02446, USA \\ ${ }^{3}$ Department of Electrical \& Computer Engineering, Boston University, 8 Saint Mary's Street, Boston, \\ MA 02215-2421, USA
}

Correspondence should be addressed to M. A. Ferrara, antonella.ferrara@na.imm.cnr.it

Received 9 June 2011; Accepted 20 July 2011

Academic Editor: Fabrice Gourbilleau

Copyright (C) 2012 M. A. Ferrara et al. This is an open access article distributed under the Creative Commons Attribution License, which permits unrestricted use, distribution, and reproduction in any medium, provided the original work is properly cited.

\begin{abstract}
The observation of stimulated Raman scattering in amorphous silicon nanoparticles embedded in Si-rich nitride/silicon superlattice structures (SRN/Si-SLs) is reported. Using a $1427 \mathrm{~nm}$ continuous-wavelength pump laser, an amplification of Stokes signal up to $0.9 \mathrm{~dB} / \mathrm{cm}$ at $1540.6 \mathrm{~nm}$ and a significant reduction in threshold power of about $40 \%$ with respect to silicon are experimentally demonstrated. Our results indicate that amorphous silicon nanoparticles are a great promise for Si-based Raman lasers.
\end{abstract}

\section{Introduction}

Raman amplification, demonstrated in the early 1970 s, is an interesting approach for optical amplification, because it is only restricted by the pump wavelength and Raman active modes of the gain medium $[1,2]$. The base phenomenon governing Raman amplification is stimulated Raman scattering (SRS), which generates vibrations in the lattice of the medium (optical phonons) and transforms the photons of the pump radiation, turning them into lower-energy ones $[1,2]$.

Recently, despite intrinsic limitations related to the nature of the bulk Si materials [3], light amplification by SRS in silicon waveguides has achieved significant results [4-8]. However, the narrowband $(105 \mathrm{GHz})$ of stimulated Raman gain in Si limits its applicability in the context of Si photonics and makes it unsuitable for its use in broadband division multiplexing (WDM) applications, unless expensive multipump schemes are implemented. Additionally, even if the Raman effect in silicon is more than 10,000 times stronger than in glass fiber, therefore, instead of kilometres of fiber, only centimetres of silicon are required, Raman amplification in $\mathrm{Si}$ is still a small effect. As a consequences, in order to build a laser based on stimulated Raman effects in $\mathrm{Si}$, very high power intensity and very low absorption losses are required.
Finally, in order to get Raman laser in silicon, the main difficulty was due to the competing nonlinear effect of two-photon absorption (TPA), which reduces the efficiency of SRS. This effect generates electron-hole pairs, which remain excited in the sample for a long time (micro-to milliseconds) and lead to strong absorption at both the pump and signal frequencies [3-8].

A breakthrough in fiber optics communications was achieved with the reduction of the water absorption peak at $1400 \mathrm{~nm}$, which opened up the available communication range to span from 1270 to $1650 \mathrm{~nm}$, corresponding to about $50 \mathrm{THz}$ bandwidth [9]. This dramatic increase in bandwidth rules out the use of existing Er-doped fiber amplifiers, leaving Raman gain as the main mechanism for future amplification needs. However, it is worth noting that concerning Raman sources, as a general rule, there is a tradeoff between gain and bandwidth in all bulk materials: linewidth is bought at the expense of peak gain. Of course, this tradeoff is a fundamental limitation towards the realization of micro-/nanosources with large emission spectra. Therefore, the investigation of new materials possessing both large Raman gain coefficients and broader spectral bandwidth than silicon is becoming mandatory in order to try to overcome the gain bandwidth tradeoff and to satisfy the increasing telecommunications demands. 
Nanostructured silicon has generated large interest in the past decades as a promising key material to establish a Sibased photonics. The low absorption coefficient makes Si$\mathrm{nc}$ attractive as active medium in waveguide structures and their third order nonlinear optical properties have been also investigated, for promising applications such as all-optical switching [10]. Concerning SRS, it is worth noting that except for a report from individual single-walled carbon nanotubes [11], the observation of SRS from GAP semiconductors nanowires [12] and from silicon nanocomposites [13, 14], we find no other evidence for this important nonlinear optic effects in nanostructured materials.

In this paper, we experimentally demonstrate a significant enhancement of the Raman gain coefficient and a reduction in threshold power using amorphous silicon nanoparticles embedded in Si-rich nitride/Silicon superlattice structures (SRN/Si-SLs) with respect to silicon [15].

\section{Sample Preparation and Its Structural and Optical Characterization}

SRN/Si-SLs samples with $450 \mathrm{~nm}$ total thickness were fabricated on Si substrates by radio frequency (RF) magnetron cosputtering from $\mathrm{Si}$ and $\mathrm{Si}_{3} \mathrm{~N}_{4}$ targets. The sputtering of the multilayer structures was performed in a Denton Discovery 18 confocal-targets sputtering system, as described elsewhere. An atomic concentration of $48 \%$ Si was measured in the deposited films with energy dispersive X-ray analysis (EDX, Oxford ISIS). The multilayer structure has been annealed using a rapid thermal annealing (RTA) furnace in $\mathrm{N} 2 / \mathrm{H} 2$ forming gas ( $5 \%$ hydrogen) for 10 minutes at $800^{\circ} \mathrm{C}$. Thermal annealing resulted in a phase separation process leading to the nucleation of amorphous Si clusters embedded in the Si nitride layers. Figure 1 shows a TEM bright-field image of our sample in cross-section, taken using a JEOL 2010 TEM operated at $200 \mathrm{KV}$. The average a-Si and SRN layer thicknesses were measured to be $21 \mathrm{~nm}$ and $26 \mathrm{~nm}$, respectively. The structure of the sample consists of 10 SRN layers and 9 amorphous $\mathrm{Si}(\mathrm{a}-\mathrm{Si}$ ) layers for a total thickness of $450 \mathrm{~nm}$. A higher magnification of a SRN layer shows the nucleation of amorphous Si nanocrystals marked by the arrows in the inset (Figure 1(b)). These amorphous clusters give rise to strong near-infrared photoluminescence with nanosecond decay dynamics at room temperature, as discussed in details elsewhere [16-19].

SRS belongs to a class of nonlinear optical processes that can be called quasiresonant. Although none of the fields is in resonance with the vibrations in the lattice of the medium (optical phonons), the difference between the pump and generated beam equals the transition frequency. This process allows a gain on an optical signal to be created, provided that the signal is propagated at the frequency of the diffused light [20]. The gain coefficient depends on scattering efficiency, the larger the spontaneous scattering efficiency of materials is, the higher the Raman gain for a given intensity is obtained.

In order to investigate Raman gain in silicon nanocomposites, the steady-state linear (no pump depletion) regime

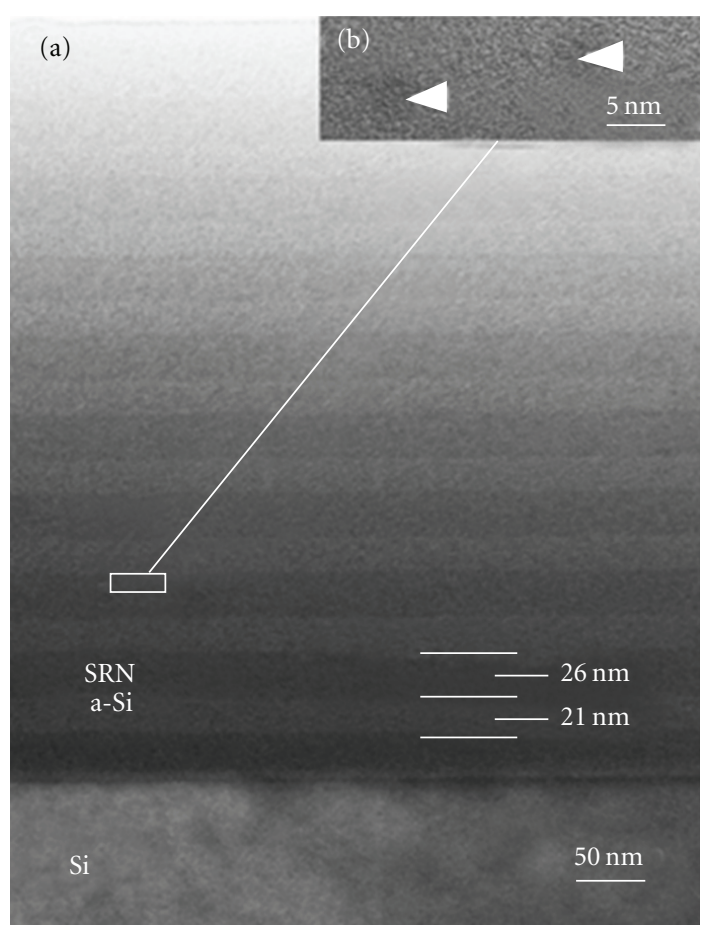

FIGURE 1: (a) Cross-sectional TEM bright-field micrograph of Sirich nitride/amorphous-Si superlattice structure consisting of 10 SRN layers and 9 a-Si layers for a total thickness of $450 \mathrm{~nm}$. (b) Higher magnification of a SRN layer, showing amorphous Si nanocrystals (approximately $2 \mathrm{~nm}$ in diameter) marked by arrows.

of SRS was considered. In this regime, the intensity of the output Stokes radiation is expressed by [20]

$$
I_{S}(L)=I_{S}(0) \cdot \exp \left(I_{P}(0) g L\right)
$$

where $I_{S 0}$ is the intensity of the input Stokes radiation (Stokes seed), $I_{S}$ is the intensity of the output Stokes radiation, $I_{P}$ is the intensity of the pump radiation, $g$ is the Raman gain coefficient, and $L$ is the effective length. Assuming no losses at the Stokes frequency, the value of the gain coefficient $g$ can be obtained by fitting (1), which is readily transformed into

$$
\mathrm{SRS}=10 \cdot \log _{10}\left(\frac{I_{S}(L)}{I_{S}(0)}\right)=4.34 \cdot g L I_{P}(0),
$$

where $I_{P}=P / A$ with $P$ as the power incident onto the sample and $A$ as the effective area of pump beam.

We note that the main physics idea behind the papers investigating SRS at nanoscale [11-14] is to suppose that this phenomena can satisfy the law valid for bulk and microstructured material. At this time, there is no reason for thinking to some different law.

Being the sample transparent to the incident light, $L$ can be taken to be equal to the thickness of the sample along the path of the incident light $(L=1.3 \mathrm{~cm})$. For calculations of the effective area, the waist of pump laser was measured by the knife-edge technique. A value of $5 \mathrm{~mm}$ was obtained.

In Figure 2 is shown the experimental setup used in order to measure SRS in SRN/Si-SLs. The pump laser is a CW 


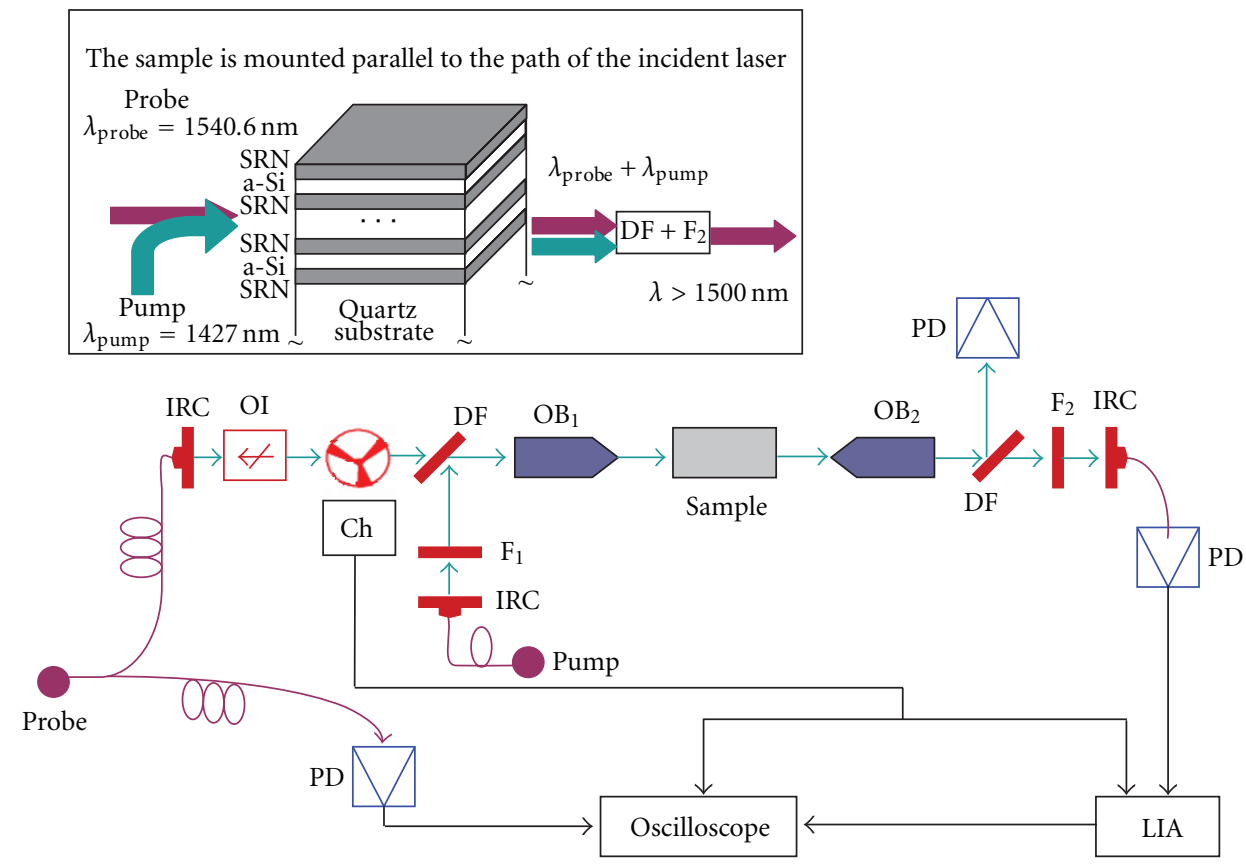

FIGURE 2: Experimental setup for SRS measurements: pump-Raman laser; IRC: infrared collimator; $\mathrm{F}_{1}$ : bandpass filter at 1427 nm; probe: ECDL external cavity diode laser (tunable); OI: optical insulator; DF: dichroic filter; $\mathrm{OB}_{1}\left(\mathrm{OB}_{2}\right)$ : microscope objective lens 50x (20x); $\mathrm{F}_{2}$ : long pass filter at $1500 \mathrm{~nm}$; Ch: Chopper; PD: optically broadband photodetector; LIA: lock-in amplifier. Black lines represent electrical connections and wiring, green lines represent free-space optical beams, and magenta lines represent optical fiber. The inset shows how the pump and the probe interact with the sample.

pump-Raman laser operating at $1427 \mathrm{~nm}$. The probe laser is a tunable external cavity diode laser (1520-1620 nm). However, the wavelength $(\lambda=1540.6 \mathrm{~nm})$ is fixed in order to get the quasiresonance. Of course, if the probe wavelength is detuned from this value, the SRS efficiency is reduced. The probe beam is split by a Y-fiber optic junctions. One of the branches is used in order to monitor probe fluctuations. The other one and the pump laser are combined on a dichroic mirror and subsequently coupled to a long working distance $50 \mathrm{x}$ infrared objective in order to be focused onto the sample. Estimated coupling losses were about $4 \mathrm{~dB}$. The transmitted signals from the sample are collected by a 20x microscope objective. In order to separate the probe from the pump, a dichroic filter and a long-pass filter were used. An optically broadband photodetector (PD) was used to collect the probe signal. The signal from the PD is demodulated by a lockin amplifier, which is externally referenced to the $180 \mathrm{~Hz}$ chopper. Each data point is averaged 1000 times before being acquired. Additionally, four measured values are averaged for each data point. The accuracy of measurement is $\pm 0.1 \mathrm{~dB}$.

The sample is mounted parallel to the path of the incident beam (as shown in the inset of Figure 2). This configuration has been used in order to point out the role of nanoparticles; therefore, the multilayer structure could be not necessary. However, in the future, we are going to explore the possible optical effect played by superlattice structure and a further enhancement on nonlinear Raman scattering is expected. In addition, we note that the sample used for TEM characterization was realised on silicon substrate, while in order to obtain the confinement of mode propagating in the multilayer, the sample used for nonlinear optical characterisation was realised on silica substrate.

In Figure 3, the peak signals are plotted as a function of the effective pump power (including the pass through the filter and objective). The maximum signal gain obtained was $0.9 \mathrm{~dB} / \mathrm{cm}$. In Figure 3, the SRS in bulk silicon is also plotted as a function of the effective pump power. Both plots in Figure 3 show an approximately linear dependence, as expected for the gain of a Raman amplifier as a function of pump power. As shown in Figure 3, SRN/Si-SLs exhibits a Raman gain significantly greater than bulk silicon. Although the estimation of the gain coefficient $g$ is not straightforward due to the uncertainty in the effective focal volume inside the sample, our data clearly demonstrate a value of $g$ which is about four time larger than the value reported for silicon [15] can be obtained in silicon nanostructures. Furthermore, our data prove a threshold power reduction of about $40 \%$ in silicon nanocrystals $\left(P_{\text {th }} \approx 150 \mathrm{~mW}\right.$ ) with respect to silicon $\left(P_{\text {th }} \approx 250 \mathrm{~mW}\right)$.

\section{Discussion}

In recent years, there is a strong interest in investigation of Raman scattering in electrons-confined and photons-confined materials. The phenomenon of strong resonant and local enhancement of visible electromagnetic (EM) radiation when incident on the surface of metallic particles and films resulting from surface plasmon resonances continues to attract significant attention for fundamental and applied interests [21]. However, the possibility of enhancement of EM 


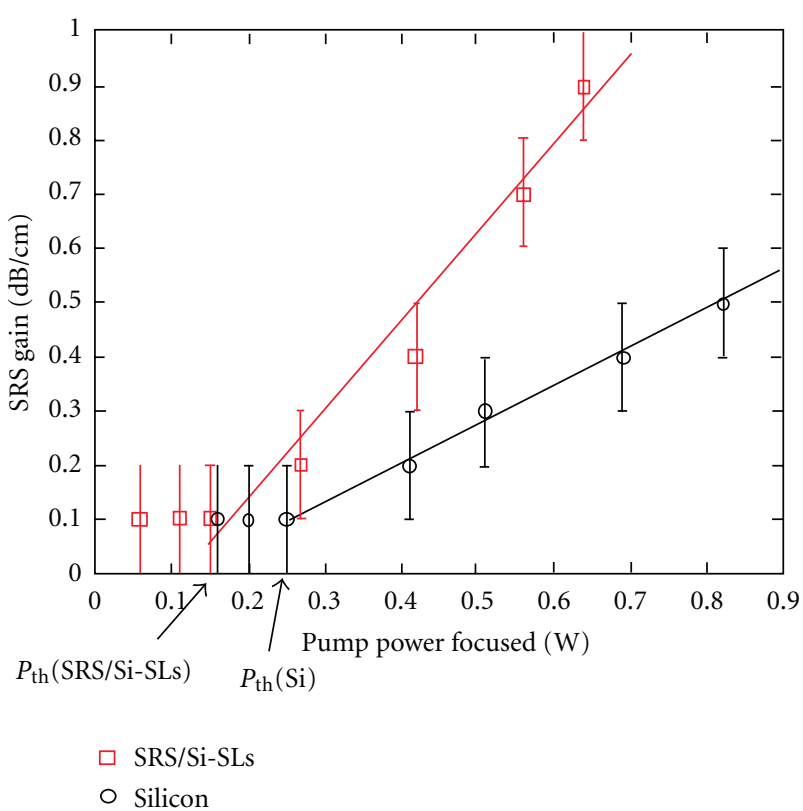

FIGURE 3: The SRS gain (amplification of the stokes signal in $\mathrm{dB} / \mathrm{cm}$ ) is plotted against the effective pump power at the sample surface both for SRN/Si-SLs ( $\square$ red) and for bulk silicon ( $\bigcirc$ black).

radiation from semiconducting and insulating materials, particularly in silicon, is noteworthy for silicon-based optoelectronic applications owing to the potential for monolithically integrating photonic technology and semiconductor electronics.

In dielectric particles, Raman scattering exhibits strong resonant effects when the wavelength of an incident field is commensurate with that of an electromagnetic eigenmode of the particle, which depends on its size and on the refractive indices of the particle and the surrounding medium [22]. This enhancement can be understood by viewing the particle as a cavity whose dimensions determine whether or not it is in resonance with either the incident or the emitted EM waves or both (i.e., double resonance).

The observations of an enhancement of about 100 of the intensity of spontaneous Raman scattering were reported in [22] from a variety of $\mathrm{Si}$ structures having submicrometer dimensions $(x \sim a / \lambda \sim 1)$, where $\lambda$ is the wavelength and $a$ is a characteristic particle dimension; for example spheres, having diameters of $\sim 0.1$ micron, were considered. As expected, the enhanced one-phonon spectra reported was a Lorentzian line shape with widths comparable with that of good-quality bulk Si, while the exploited resonances were corresponding to electromagnetic cavity modes with at least one optical wavelength resonating within the particle.

Strong enhancement $\left(\sim 10^{3}\right)$ of the spontaneous Raman scattering was reported from individual silicon nanowires and nanocones as compared with bulk Si [23]. The observed enhancement was diameter, excitation wavelength, and incident polarization state dependent. The observed increase in Raman-scattering intensity with decreasing diameter in this system was explained in terms of structural resonances in the local field similar to Mie scattering from dielectric spheres.

Before concluding, it is worth noting that, in our previous papers [24, 25], some advantages of $\mathrm{Si}$-nc with respect to silicon were pointed out. Experimental results proving spontaneous Raman scattering in Si-nc at the wavelength of interest for telecommunications $(1.54 \mathrm{~mm})$ were reported in [24]. According to phonon confinement model, in [25], two significant improvement of Raman approach in silicon quantum dots with respect to silicon were reported: the broadening of spontaneous Raman emission and the tuning of the Stokes shift. Considering silicon quantum dots having crystal size of $2 \mathrm{~nm}$, a significant broadening of about $65 \mathrm{~cm}^{-1}$ and a peak shift of about $19 \mathrm{~cm}^{-1}$ were obtained. Taking into account such results, more than the half of C-band telecommunication (width $=146 \mathrm{~cm}^{-1}$ ) could be covered using silicon quantum dots, without implementing the multipump scheme. Finally, prospects of Raman amplifier in silicon nanostructure were discussed [25]. The possibility to enhance the Raman gain coefficient and to reduce two-photon absorption, at the same time, in silicon quantum dot was addressed too [25].

Therefore, our previous results on the broadening of the Raman gain spectra [24,25] combined with the present observation of enhanced Raman gain lead us to conclude that the traditional tradeoff between gain and bandwidth could be overcome in low-dimensional materials.

In addition, in our previous papers $[13,14]$, the stimulated Raman effect in silicon nanocomposite material has been investigated. We note that there is a fundamental difference between the previous experiment and the ones reported in this paper. Concerning the previous experiment, being the diameter of particles dispersed in silica matrix of about 0.1 micron, the enhancement of SRS was due to a photons confinements effect. Whereas, in this experiment, being the particle dimension of about $2 \mathrm{~nm}$, the phonon confinement effect is significant; therefore, we suggest that enhancement explanation has to be sought out in the framework of matter confinement and related to the enhancement of the thirdorder nonlinear susceptibility in small Si clusters (approximately $2 \mathrm{~nm}$ in diameter) that nucleate in a high density inside silicon-rich nitride materials. However, the structure of the SRN/Si interfaces, the stoichiometric material disorder, and the cluster dimensionality are also important parameters that are expected to significantly influence Raman amplification, a theoretical understanding of their respective roles remains to be established.

\section{Conclusions}

In this paper, we experimentally demonstrate that amorphous Si nanocrystals can provide larger gain values and a significant reduction in threshold power with respect to bulk Si devices. In addition, we prove that the tradeoff between gain and bandwidth in all bulk materials can be overcome in nanostructured materials.

Further studies will be carried on in the near future addressing the physical origin of enhanced Raman gain in nanostructured and superlattice-based Si systems. We believe 
that our experimental results open the way to the investigation of important issues for fundamental and applied interests, for example, the investigation of nonlinear optical phenomena (SRS) at nanoscale and fabrication of more efficient Raman lasers compatible with Si technology.

\section{References}

[1] M. N. Islam, "Raman amplifiers for telecommunications," IEEE Journal on Selected Topics in Quantum Electronics, vol. 8, no. 3, pp. 548-559, 2002.

[2] A. Mori, H. Masuda, K. Shikano, and M. Shimizu, "Ultrawide-band tellurite-based fiber Raman amplifier," Journal of Lightwave Technology, vol. 21, no. 5, pp. 1300-1306, 2003.

[3] B. Jalali, V. Raghunathan, D. Dimitropoulos, and O. Boyraz, "Raman-based silicon photonics," IEEE Journal on Selected Topics in Quantum Electronics, vol. 12, no. 3, pp. 412-421, 2006.

[4] R. Dekker, N. Usechak, M. Först, and A. Driessen, "Ultrafast nonlinear all-optical processes in silicon-on-insulator waveguides," Journal of Physics D, vol. 40, no. 14, pp. R249-R271, 2007.

[5] O. Boyraz and B. Jalali, "Demonstration of a silicon Raman laser," Optics Express, vol. 12, no. 21, pp. 5269-5273, 2004.

[6] H. Rong, A. Liu, R. Jones et al., "An all-silicon Raman laser," Nature, vol. 433, no. 7023, pp. 292-294, 2005.

[7] H. Rong, R. Jones, A. Liu et al., "A continuous-wave Raman silicon laser," Nature, vol. 433, no. 7027, pp. 725-728, 2005.

[8] H. Rong, S. Xu, Y. H. Kuo et al., "Low-threshold continuouswave Raman silicon laser," Nature Photonics, vol. 1, no. 4, pp. 232-237, 2007.

[9] C. Rivero, K. Richardson, R. Stegeman et al., "Quantifying Raman gain coefficients in tellurite glasses," Journal of NonCrystalline Solids, vol. 345-346, pp. 396-401, 2004.

[10] S. Hernández, P. Pellegrino, A. Martínez et al., "Linear and nonlinear optical properties of $\mathrm{Si}$ nanocrystals in $\mathrm{Si} \mathrm{O} 2$ deposited by plasma-enhanced chemical-vapor deposition," Journal of Applied Physics, vol. 103, no. 6, Article ID 064309, 2008.

[11] B. P. Zhang, K. Shimazaki, T. Shiokawa, M. Suzuki, K. Ishibashi, and R. Saito, "Stimulated Raman scattering from individual single-wall carbon nanotubes," Applied Physics Letters, vol. 88, no. 24, pp. 241101-241103, 2006.

[12] J. Wu, A. K. Gupta, H. R. Gutierrez, and P. C. Eklund, "Cavity-enhanced stimulated raman scattering from short GaP nanowires," Nano Letters, vol. 9, no. 9, pp. 3252-3257, 2009.

[13] L. Sirleto, M. A. Ferrara, G. Nicotra, C. Spinella, and I. Rendina, "Observation of stimulated Raman scattering in silicon nanocomposites," Applied Physics Letters, vol. 94, no. 22, Article ID 221106, 2009.

[14] M. A. Ferrara, L. Sirleto, G. Nicotra, C. Spinella, and I. Rendina, "Enhanced gain coefficient in Raman amplifier based on silicon nanocomposites," Photonics and NanostructuresFundamentals and Applications, 2010.

[15] J. M. Ralston and R. K. Chang, "Spontaneous-ramanscattering efficiency and stimulated scattering in silicon," Physical Review B, vol. 2, no. 6, pp. 1858-1862, 1970.

[16] L. Dal Negro, R. Li, J. Warga, and S. N. Basu, "Sensitized erbium emission from silicon-rich nitride/silicon superlattice structures," Applied Physics Letters, vol. 92, no. 18, Article ID $181105,2008$.

[17] L. Dal Negro, J. H. Yi, J. Michel et al., "Light emission efficiency and dynamics in silicon-rich silicon nitride films," Applied Physics Letters, vol. 88, no. 23, Article ID 233109, 2006.
[18] L. Dal Negro, J. H. Yi, L. C. Kimerling, S. Hamel, A. Williamson, and G. Galli, "Light emission from silicon-rich nitride nanostructures," Applied Physics Letters, vol. 88, no. 18, Article ID 183103, 2006.

[19] R. Li, J. R. Schneck, J. Warga, L. D. Ziegler, and L. Dal Negro, "Carrier dynamics and erbium sensitization in silicon-rich nitride nanocrystals," Applied Physics Letters, vol. 93, no. 9, Article ID 091119, 2008.

[20] Y. R. Shen and N. Bloembergen, "Theory of stimulated brillouin and raman scattering," Physical Review, vol. 137, no. 6A, pp. A1787-A1805, 1965.

[21] S. Kawata, Y. Inouye, and P. Verma, "Plasmonics for near-field nano-imaging and superlensing," Nature Photonics, vol. 3, no. 7, pp. 388-394, 2009.

[22] D. V. Murphy and S. R. J. Brueck, "Enhanced Raman scattering from silicon microstructures," Optics Letters, vol. 8, pp. 494496, 1983.

[23] L. Cao, B. Nabet, and J. E. Spanier, "Enhanced Raman scattering from individual semiconductor nanocones and nanowires," Physical Review Letters, vol. 96, no. 15, Article ID 157402, pp. 1-4, 2006.

[24] L. Sirleto, V. Raghunatan, A. Rossi, and B. Jalali, "Raman emission in porous silicon at $1.54 \mu \mathrm{m}$," Electronics Letters, vol. 40, no. 19, pp. 1221-1222, 2004.

[25] L. Sirleto, M. A. Ferrara, I. Rendina, and B. Jalali, "Broadening and tuning of spontaneous Raman emission in porous silicon at $1.5 \mu \mathrm{m}, "$ Applied Physics Letters, vol. 88 , no. 21 , Article ID $211105,2006$. 


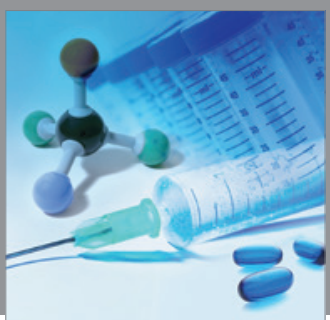

International Journal of

Medicinal Chemistry

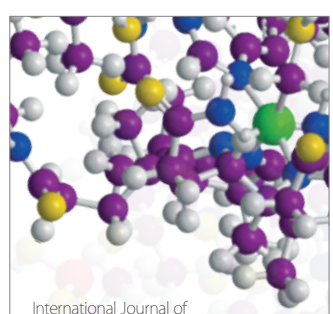

Carbohydrate Chemistry

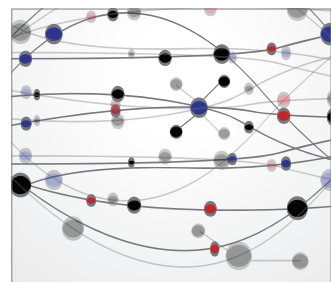

The Scientific World Journal
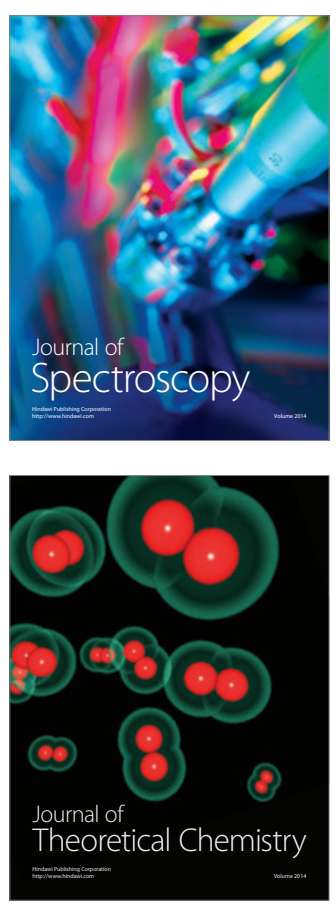
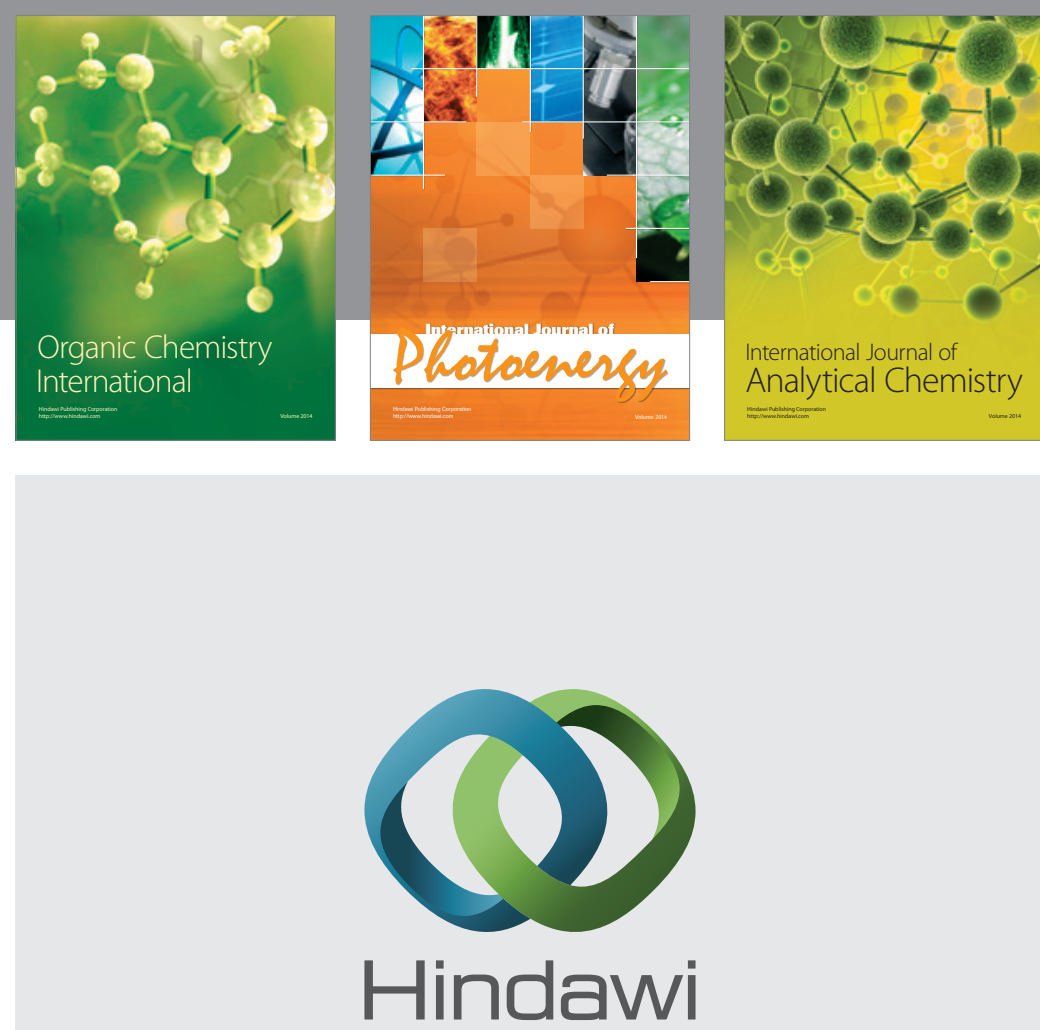

Submit your manuscripts at

http://www.hindawi.com
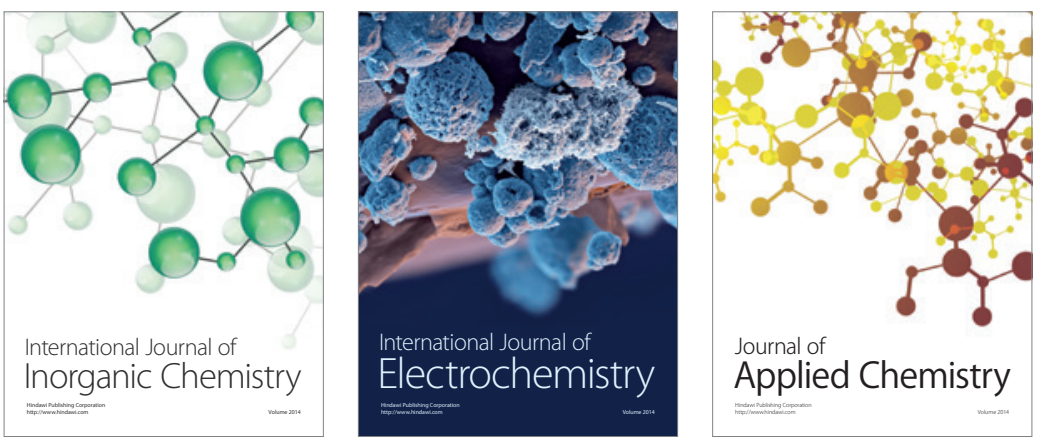

Journal of

Applied Chemistry
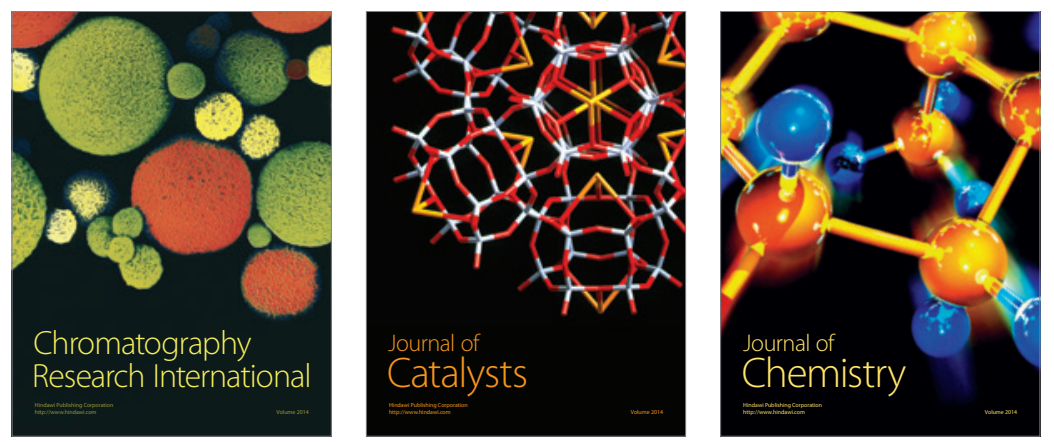
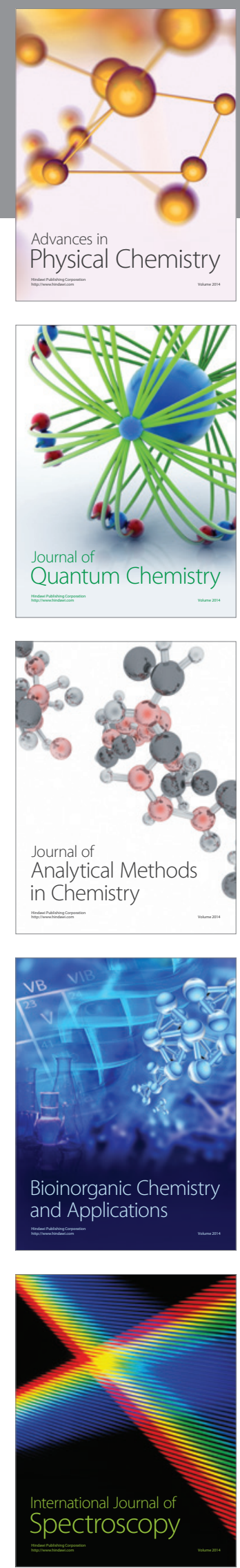